\title{
Tecnologías de la información y la comunicación: bases del aprendizaje significativo en matemáticas en estudiantes universitarios.
}

\section{Information and Communication Technologies: bases of significant learning in mathematics in university students.}

Marco Antonio Gavilanes Sagñay. ${ }^{1}$, Willian Geovanny Yanza Chávez. ${ }^{2}$, Alfredo Rodrigo Colcha Ortiz. ${ }^{3}$ \& Efraín Velasteguí López ${ }^{4}$

\begin{abstract}
The development and implementation of new information technologies in the educational context have progressed in correspondence with changes in teachinglearning methods. In contradiction with the above, the pedagogical praxis based on the use of traditional methods for teaching - learning Mathematics still persist in the different educational settings and levels, generating low levels of performance, boredom and even the apathy of students when learning it. In accordance with what has been pointed out, this article aims to: reflect on the importance and impacts of the teaching-learning process of Mathematics, the intrinsic relationship that must exist between student-teachers and the educational means to be used, within these the proper application of ICTs, as a basic premise for achieving significant learning. Based on a search methodology, documentary reviews, inquiry and reflections on previous studies, two essential aspects are confirmed as results: the first related to the confirmation of the importance of this trilogy in the acquisition of significant learning in students and the second already our way of seeing with high significance: the need to integrate various methodological perspectives that lead to the construction of knowledge, both for

\footnotetext{
${ }^{1}$ Escuela Superior Politécnica de Chimborazo, Facultad de Administración de Empresas, Chimborazo Ecuador, magavilanes@yahoo.es

${ }^{2}$ Escuela Superior Politécnica de Chimborazo, Facultad de Administración de Empresas, Chimborazo Ecuador, willianyanza@hotmail.com

${ }^{3}$ Gobierno Autónomo Descentralizado Municipal del Cantón Riobamba., Chimborazo Ecuador, colchaa@gadmriobamba.gob.ec

${ }^{4}$ Ciencia Digital Editorial, Ambato Ecuador, luisefrainvelastegui@cienciadigital.org
} 
teachers and students, through the significant learning model through the application of Information and Communication Technologies, all of which will favor the development of positive and critical attitudes towards mathematics by students.

Key words: New Information Technologies, Mathematics, meaningful learning.

\section{Resumen}

El desarrollo e implementación de las nuevas tecnologías de la información en el contexto educativo, han progresado en correspondencia con los cambios en los métodos de enseñanza-aprendizaje. En contradicción con lo expuesto aún persisten en los diferentes escenarios y niveles educativos la praxis pedagógica basada en la utilización de métodos tradicionales para la enseñanza - aprendizaje de la Matemática, generándose bajo nivel de desempeño, aburrimiento y hasta la apatía de los estudiantes al aprenderla. En correspondencia con lo señalado este artículo tiene como objetivo: reflexionar sobre la importancia e impactos que tiene en el proceso de enseñanza aprendizaje de las Matemáticas la relación intrínseca que debe darse entre estudiantesdocentes y los medios educativos a emplear, dentro de estos la aplicación adecuada de las TICs, como premisa básica para el logro de aprendizajes significativos. Basado en una metodología de búsqueda, revisiones documentales, indagación y reflexiones sobre estudios precedentes, se confirma como resultados dos aspectos esenciales: el primero relacionado con la confirmación de la importancia de esta trilogía en la adquisición de aprendizajes significativos en los estudiantes y el segundo y a nuestro modo de ver de elevada significatividad: la necesidad de integrar diversas perspectivas metodológicas que conlleven a la construcción de conocimientos, tanto de los docentes como de los estudiantes, a través del modelo de aprendizaje significativo mediante la aplicación de las Tecnologías de la Información y Comunicación, todo lo cual va a favorecer el desarrollo de actitudes positivas y críticas hacia las matemáticas por parte de los estudiantes.

Palabras claves: Nuevas Tecnologías de la Información, Matemática, aprendizaje significativo.

\section{Introducción}

La teoría del aprendizaje significativo planteada por David P. Ausubel en 1963, trae a locación la alternativa de un modelo de enseñanza- aprendizaje nuevo, que enmarca como aspecto fundamental la sistematicidad del aprendizaje cognitivo, contextualizando la incorporación de los nuevos conceptos en articulación directa con los conocimientos previos, que de por sí ya el estudiante posee de manera esencial, con la finalidad de que tengan significado.

Es justamente en este proceso que se da la interacción de los tres elementos que conforman el proceso de enseñanza-aprendizaje: estudiantes, profesor y los materiales educativos utilizados; todos los cuales desempeñan diferentes roles dentro del proceso de enseñanza-aprendizaje, pero independientemente de los roles que ocupan se mantienen estrechamente vinculados. En el caso de los sujetos: estudiantes y profesor, ambos lo hacen de manera activa, correspondiéndole al profesor, como guía, evaluar si los objetivos planteados fueron cumplidos conforme a lo planificado. 
En este marco, la implementación en el contexto educativo de las Nuevas Tecnologías de la Información, más conocidas como las TIC, han progresado en el decursar histórico en correspondencia con los cambios en los métodos y estilos de enseñanza-aprendizaje, motivando el desarrollo de manera creciente de capacidades, aptitudes físicas y mentales en los estudiantes, favoreciendo considerablemente su desarrollo cognitivo.

En el caso específico de la aplicación de las TIC en el proceso de aprendizaje de las matemáticas, la efectividad de su aplicación no solo es percibida por su beneficio para la capacitación certificada y técnica de los estudiantes, sino además que hay razones propias de esta materia que justifican su enseñanza con miras muy claras hacía la preparación cognitiva con vistas a la formación como futuro profesional; dentro de estos los aspectos motivacionales, ocupan un lugar importante dado que tienen un elevado peso en las elecciones vocacionales de los estudiantes. Cabe recalcar que depende mucho de la manera como se enseñe la materia, que se facilite la atracción a la misma, independientemente a los contenidos que enmarca y su adecuada integración curricular.

Es por ello que es fundamental la forma en que se direccione el proceso de enseñanzaaprendizaje de las matemáticas en los diferentes niveles de enseñanza, dado que la aplicación de las TIC en las matemáticas, a través del uso de herramientas tecnológicas, debe estar claramente vinculadas a la propia elaboración del conocimiento matemático.

Una vez que el docente verifique a través de su propia experiencia la potencialidad de las tecnologías a su disposición, la utilidad de su uso y la posible dificultad que el grado de asimilación del contenido le depare al estudiante, podrá propiciar las condiciones para un aprendizaje significativo de las ciencias matemáticas, mediante la aplicación de las TICs.

Buscar alternativas factibles que respondan a estas nuevas exigencias educacionales constituye un gran reto, dado que aún existen muchas cuestiones sin solución en relación a la consecución de un aprendizaje significativo en las matemáticas a través de la aplicación de las TIC. En este orden de ideas el objetivo de este artículo, esta' centrado en reflexionar sobre la importancia e impactos que tiene en el proceso de enseñanza -aprendizaje de las Matemáticas la relación intrínseca que debe darse entre estudiantes-docentes y los medios educativos a emplear, dentro de estos la aplicación adecuada de las TICs, como premisa básica para el logro de aprendizajes significativos en los estudiantes universitarios.

\section{Aprendizaje significativo}

La propia concepción de este modelo, magnifica las acciones a realizarse en el aula, por los estudiantes bajo la certera guía del docente, ya que supone, como punto de partida en ellos un conocimiento existente que servirá de base al otro nuevo conocimiento que se incorporará, conforme a como expresó (Ausubel,1976, pág. 6): "Si tuviese que reducir toda la psicología educativa a un solo principio, enunciaría éste: de todos los factores que influyen en el aprendizaje, el más importante consiste en lo que el alumno ya sabe. Averígüese esto, y enséñese consecuentemente". 
Y es justamente que en este proceso, la interacción de los tres elementos que lo conforman: estudiantes, profesor y los materiales educativos empleados, juegan sus respectivos importantes roles y en el caso de los sujetos: estudiantes y profesor, ambos de manera activa, correspondiéndole al profesor, como guía, el de evaluar si los objetivos planteados del aprendizaje fueron cumplidos conforme a lo planificado, al respecto coincidimos con (Gowin, 1981, pág. 81), cuando expresa "la enseñanza se consuma cuando el significado del material que el alumno capta es el significado que el profesor pretende que ese material tenga para el alumno."

La interacción de estos tres elementos es esencial y constituyen la piedra angular en este proceso de aprendizaje significativo, en tal sentido compartimos con (Rodríguez, 2008; Rodríguez, Caballero y Moreira, 2010), cuando expresaron que "La aportación esencial de Gowin es el establecimiento de una interacción tríadica profesor/alumno/materiales educativos del currículum tendente a compartir significados, sin la que de ningún modo se obtendría un aprendizaje significativo".

No obstante, aún existe mucha confusión en la aplicación del aprendizaje significativo en los centros educativos, debido a que se potencia el mismo como si lo fuera, cuando realmente se ha banalizado su utilización, ignorándose su real significado, la fundamentación teórica que lo sostiene y su proceso evolutivo. Al respecto aceptamos el criterio expuesto por (Rodríguez, 2004 a y b, 2008) al expresar que "para delimitar adecuadamente su sentido y para aplicarlo de manera más efectiva, coherente con su fundamentación teórica, es pertinente revisar lo que podemos denominar los "noes" del aprendizaje significativo, que se exponen a continuación: 1) No es posible desarrollar aprendizajes significativos si no se cuenta con una actitud significativa de aprendizaje; sin ésta, el aprendizaje que se produce será repetitivo y mecánico. 2) No se genera tampoco aprendizaje significativo si no están presentes las ideas de anclaje pertinentes en la estructura cognitiva del aprendiz."

En este orden, ocurre algo muy importante y es precisamente poder precisar y clarificar que no siempre que el estudiante quiera aprender, significa que estamos en presencia de que se vaya a dar un aprendizaje significativo, ello no es suficiente; para que realmente lo sea, se hace necesario otra principal condición y es la referida a contar con un material educativo potencialmente significativo, ello implica que si el docente no realiza su labor de manera adecuada con el mismo, el estudiante no aprenderá de forma significativa.

El aprendizaje significativo trae consigo el crecimiento cognitivo del estudiante, que al unísono también incluye un crecimiento afectivo, en la medida en que se motiva y adecua su predisposición hacia nuevos aprendizajes. Al mismo tiempo, ofrece al docente el motivo para que comprenda el proceso del conocimiento, y a su vez le proporciona las estrategias de acción adecuadas dentro del proceso enseñanza-aprendizaje que genere en sus estudiantes un aprendizaje significativo.

Lo anterior fundamenta el papel trascendental que tiene el docente en este proceso, de su accionar consciente y de su actitud proactiva en direccionar de manera consistente y efectiva la generación de un aprendizaje significativo en sus estudiantes. En relación a ello compartimos 
con (Moreira, 2012) al expresar que "La facilitación del aprendizaje significativo depende mucho más de una nueva postura docente, de una nueva directriz escolar, que de nuevas metodologías, incluso las modernas tecnologías de información y comunicación."

\section{La aplicación de las TIC en el contexto educativo.}

En la era de la globalización, el uso de las Tecnologías de la Información y la Comunicación constituye una de las aplicaciones fundamentales en el campo de la educación, lo que ha influenciado de manera significativa en la creación de tecnologías educativas en este ámbito, incorporándose a nuestro quehacer educativo diario en las aulas, aspecto que ha sido resaltado por Landeau (2012) al definir las TIC como aquellas: “ Herramientas computacionales e informáticas, que procesan, sintetizan, reivindican y presentan información representada de la forma más renovada. Este recurso es incuestionable, ya que forma parte de nuestra cultura tecnológica, nos rodea y con la cual debemos convivir. (p. 130)".

Paralelamente a ello, el sistema educativo tiene ante sí, en la actual sociedad de la información y el conocimiento, el enorme desafío de formar estudiantes capaces y con un alto nivel de preparación profesional lo que implica la materialización de propuestas y entornos educativos nuevos mediante la creación de un entorno propicio en el aula que posibilite el uso y la aplicación de las Tecnologías de la Información y la Comunicación (TIC), por parte de los estudiantes, que les permita aprender, comunicar y desarrollar sus capacidades creativas. Esto solo puede lograrse mediante la aplicación del aprendizaje significativo.

No obstante la utilización de las Tecnologías de la Información y la Comunicación como herramientas de apoyo y contribución a las materias educativas y en especial a las matemáticas, transcurre por un camino no exento de dificultades y de trabas en la consecución de los verdaderos objetivos que se desean alcanzar, ya que en muchas ocasiones el docente y el estudiante hacen prevalecer el aprendizaje de las propias tecnologías por sobre el conocimiento de los fundamentos de la materia en cuestión, cuando realmente debe realizarse un proceso paralelo de obtención de los conocimientos que no vaya en detrimento de ni de la formación del conocimiento ni del desarrollo propio de las habilidades con las tecnologías.

En este ámbito, en ocasiones ocurre lo contrario, en que se reduce la aplicación de las tecnologías, al no dejar en plena libertad de uso de los software a los estudiantes en sus actividades de investigación, o sea se les cierra la ampliación de su abanico de utilización de las tecnologías, forzándolos a depender del uso de determinados software para su investigación, cuando los docentes debieran permitir, siempre bajo su certera guía, la utilización de los softwares de manera libre a los estudiantes de manera que propicien el acceso a la investigación.

Estos aspectos han sido recogido en estudios realizados al respecto por diversos investigadores, en relación a ello, coincidimos con Chiluiza García (2004, p. 9) quien respecto al uso de las Tecnologías de la Información y Comunicación expresó "estos estudios concluyen que a la par de la construcción de conocimiento, se desarrollan habilidades altas del pensamiento, como la 
capacidad de usar estrategias en la solución de problemas, pensamiento crítico y creatividad; y, que coinciden con las requeridas por la UNESCO".

En este escenario de la utilización de las Tecnologías de la Información y Comunicación en el ámbito de la educación, pueden producirse otras posibilidades de comunicación educativaformativa, que hasta el momento estaban supeditados al actual modelo cultura en uso, constituyendo este otro riesgo que debe salvarse debido a que tampoco deben eliminarse completamente algunas actividades como son las conferencias o clases magistrales, pero debieran ser estas adecuadas a este contexto virtual.

Lo cierto es que estaríamos cometiendo una gran equivocación sino somos capaces de explorar todas las posibilidades que nos ofrecen las TIC, siempre sin dejar de aplicar las buenas prácticas educativas, dado que pudiéramos distanciarnos de la realidad del estudiante para focalizarnos en una dinámica de carácter estático por excelencia, lo que resultaría en la no obtención de los resultados esperados por el uso de las tecnologías.

Compartimos con Pérez, (2001, p. 5) cuando confirma que "Es en este nuevo contexto en el que las redes telemáticas, las Nuevas Tecnologías de la Comunicación puestas al servicio de la Educación pueden generar otras posibilidades de comunicación educativo-formativa que hasta este momento tenía una mayor dependencia del modelo cultural vigente. Sin embargo, no es menos cierto que nuestra dependencia de este modelo aún es tan importante que corremos el riesgo de trasladar a las nuevas rutinas del antiguo, lo que puede condicionar definitivamente su éxito".

Como expresábamos anteriormente, la implementación de las nuevas tecnologías en el contexto educativo, han progresado en correspondencia con los cambios en los métodos de enseñanza-aprendizaje, incorporando de manera creciente capacidades y aptitudes físicas y mentales en los estudiantes que los ha influenciado considerablemente en su desarrollo cognitivo, para prepararlos de una manera más efectiva en su desarrollo profesional futuro en una sociedad con un elevado nivel de tecnificación, ello ha sido aseverado por Fainholc (2012) cuando estableció que: "La tecnología educativa debe responder a las necesidades específicas de las sociedades en las cuales habrá de funcionar, debe ser pertinente, debe tener presencia en las políticas públicas, adaptarse a los sistemas sociales y culturales, a los intereses lingüísticos de los grupos receptores participantes. (p. 16)".

De esta manera, los docentes han incursionado en un nuevo horizonte de oportunidades para el desarrollo de su práctica pedagógica a través de la aplicación de las TIC como otro medio educativo más en el proceso de enseñanza-aprendizaje, influyendo en las distingas vertientes de este proceso, tales como: permitiéndoles promocionar y favorecer la actitud participativa y creadora de los estudiantes, elevando la auto-enseñanza del aprendizaje interactivo, la formación cognitiva a distancia así como el surgimiento de nuevas metodologías engendradoras de conocimientos como objetos de investigación en el crecimiento cognitivo 
asistido por computadoras, lo que ha generado una profunda transformación en el proceso de enseñanza-aprendizaje, traspasando el rol de protagonismo al estudiante.

\section{Las TICs aplicadas a las matemáticas.}

En lo que a ello respecta, la efectividad de la aplicación de las TIC, en el proceso de enseñanzaaprendizaje de las matemáticas es un aspecto que debe ser bien determinado, dado que el objetivo a lograr es incorpórale al estudiante el conocimiento propiamente de la matemática, auxiliándose de las herramientas tecnológicas, de manera que los mismos obtengan la preparación cognitiva requerida con vistas a su futuro profesional, es por eso que, es fundamental la forma en que se direccione el proceso de enseñanza- aprendizaje de la asignatura en los niveles de enseñanza, a manera de impedir, que el interés del estudiante se desplace hacia el conocimiento de estas herramientas sin aplicarlas precisamente al desarrollo cognitivo de la matemática, constituyendo este un elemento de impacto significativo de la vinculación de las TIC con esta ciencia, que a futuro tendrán los estudiantes.

Es por ello que se hace muy necesario precisar la manera de cómo con esta vinculación matemáticas-TIC se desarrollará el proceso de enseñanza-aprendizaje, dado que debe ser enseñada a los estudiantes de manera que realmente contribuya a la formación integral de ese futuro profesional, concibiendo la aplicabilidad de las TIC como un fortalecimiento de su capacidad intelectual en las matemáticas, como una forma de coadyuvar al desarrollo cognitivo e integral del estudiante.

Esto implica que en este proceso de aprendizaje también deba tenerse en cuenta aspectos motivacionales, dado que un elevado peso en las elecciones vocacionales de los estudiantes depende de la manera como se enseñe la materia, que facilite la atracción a la misma, independientemente a los contenidos que enmarca. En esta dirección es fundamental el rol del docente. Al respecto coincidimos con (Castellanos y Cervantes, 2017), citado por (Grisales, 2018) cuando expresa "En segunda instancia está la relación dialéctica entre el docente y el estudiante, en la cual el primero dista del segundo en metodologías, motivaciones y necesidades de formación específicas".

No obstante a todo lo expuesto, el área de estudios de las matemáticas constituye una de las que más se ha retrasado en incorporar la utilización de las TIC en su proceso de enseñanzaaprendizaje, utilizándose en un elevado grado los métodos tradicionales y la implementación de procesos de carácter mecánicos, que no promueven en los estudiantes aportaciones significativas de los conocimientos estudiados en su futuro profesional, dado principalmente a que se enfrentan a un escenario muy diferente al supuesto en los cuales se basaron las concepciones aprendidas durante el proceso de enseñanza-aprendizaje recibido.

En este orden de idea compartimos con (Vega, et al. 2015) cuando afirma "La matemática sin embargo, ha sido uno de los campos del saber que más ha tardado en incorporar estas estrategias y en dar un salto importante hacia la utilización de las TIC como apoyo a los procesos de aprendizaje, siendo todavía frecuente el uso de metodologías tradicionales y la realización de procesos mecánicos, descontextualizados y que no generan reflexiones 
importantes en los estudiantes sobre la utilidad que tienen los conceptos estudiados en su formación académica y en su vida cotidiana".

\section{La aplicación de las TICS y el rol del docente que imparte las matemáticas}

Desde este punto de vista, el docente de matemáticas tiene el compromiso de ofrecer al estudiante situaciones de aprendizaje que recaben su participación en planteamientos de problemas resultados de sus experiencias cognitivas de manera que les sea atractivos, apreciando y respetando los esquemas y procedimientos que el estudiante elija para afrontar los problemas o cuestiones planteadas. A su vez, el docente, como orientador de la tarea, por ningún motivo debe intentar suplantar al estudiante en sus esfuerzos de solución, sino que contrariamente, debe estimularlo de manera que persista y siga adelante con sus ideas preconcebidas para solucionar el problema. Cuando el estudiante ya cuente con una solución determinada, la intervención del docente debe centrarse en la orientación el proceso formativoreflectivo del estudiante ante la actividad realizada con la finalidad de guiar el proceso de trasferencia, generalización y desarrollo de los conocimientos adquiridos.

Es en este contexto podemos aseverar que la aplicación de las TIC incorpora transformaciones relevantes para los docentes, no solo por el contenido que debe ser enseñado a los estudiantes sino también en la preparación metodológica y cognitiva que deben alcanzar para enfrentar de manera adecuada, los procedimientos y prácticas que se generan en las aulas virtuales de esta asignatura. Pero ello es recompensado por el rompimiento de los comportamientos que se producen en los estudiantes, en comparación con los que generalmente se presenta, por el nivel alcanzado al utilizar los métodos de aprendizaje convencionales, tales como el bajo nivel de desempeño, aburrimiento y hasta la antipatía hacia la asignatura.

Todas estas irregularidades y desviaciones son superadas al incorporarse nuevas formas de aprendizaje, las que están basadas en la investigación, dando protagonismo a los estudiantes por su propio aprendizaje, anclando las ideas requeridas en la estructura cognitiva de los mismos, todo a través del logro de un aprendizaje significativo y que a su vez se encuentren incluidas en el currículo de la asignatura, conforme a lo que expresan varios autores entre los cuales podemos citar (Grisales, 2018), al señalar que "para lograr aprendizajes significativos de la matemática utilizando recursos tecnológicos es necesario articular en los currículos de formación las competencias comunicativas y tecnológicas, no solo en los estudiantes sino también en los docentes quienes deben transformar los métodos tradiciones de enseñanza de esta área."

\section{Los medios y herramientas de las TICs aplicados al proceso de aprendizaje de matemáticas.}

Como se ha explicado con anterioridad la aplicación de las TIC en el proceso de aprendizaje de las matemáticas, requiere además de los medios propiamente tecnológicos, contar con los soportes propiamente pedagógicos, disciplinares y contextuales, dado que estos elementos participan en el desenvolvimiento y crecimiento de manera recurrente durante todo el proceso de aplicación de las mismas en la formación de la asignatura. En este escenario, importancia 
trascendental reviste el uso y aprovechamiento de los Objetos Virtuales de Aprendizaje (OVA), los mismos deben estar realmente apegado al componente propio de la matemática, convirtiéndose en las nuevas herramientas del aula, apoyados, por supuesto, por el conocimiento de las TIC para su implementación, así como la creación de los Entornos Virtuales de Aprendizajes (EVA), en el cual se desarrollan los procesos de incorporación de habilidades y conocimientos a través de sistemas tecnológicos incorporados, conforme a lo planteado por (Vega, et al. 2015) quien planteó que "el componente formal de las matemáticas es fundamental en el desarrollo de los OVA y no puede ser minimizado por factores como la estética y la flexibilidad de un EVA" (p. 175).

En esta revolución de utilizar las herramientas que provee las TIC en el aprendizaje de las matemáticas es importante de igual forma, comprender que no todos los medios tecnológicos son convenientes utilizarlos en su proceso de aprendizaje y le corresponderá al docente la responsabilidad de valorar la factibilidad del uso de la aplicación de los mismos.

Por otra parte precisamente el docente es el responsable de emitir su dictamen acerca de la efectividad de los OVA y el grado de correspondencia en su uso, que favorezca el aprendizaje de los conceptos y saberes estudiados durante el proceso de enseñanza-aprendizaje de la asignatura. En esta idea (Triana, et al. 2016), afirmó que "Por otro lado, es clave entender que no cualquier tipo de recurso es apropiado para el aprendizaje efectivo de ciertos conceptos matemáticos y es el profesor quien debe evaluar en primera instancia la potencialidad de este recurso. Para esto, el docente debe contar con los criterios adecuados que le permitan valorar la eficiencia y la eficacia de los OVA y qué tanto se propicia el aprendizaje de los conceptos estudiados con su uso".

O sea, la aplicación de las TIC en las matemáticas, en su uso como herramientas tecnológicas, debe estar claramente vinculadas a la propia elaboración del conocimiento matemático, una vez que el docente verifique a través de su propia experiencia la potencialidad de las tecnologías a su disposición en la utilidad de su uso y en la posible dificultad que el grado de asimilación del mismo le depare al estudiante. Esta elaboración del conocimiento a aplicar en el proceso de aprendizaje, debe formar parte del currículo respectivo de la asignatura, de manera que no resulte de una acción individualista de un docente. Además en la actualización del currículo es recomendable incorporar el uso de las herramientas de las Tecnologías de la Información y Comunicación que serán usadas por los estudiantes, entre las competencias que deberán desarrollar en el proceso de aprendizaje.

Al respecto coincidimos con (Villa, et al. 2013) al afirmar que "Bajo esta mirada, los recursos tecnológicos en el salón de clases deben ser valorados por el alto potencial que tienen para la construcción del conocimiento matemático, cuya construcción empieza en el momento en el que el mismo docente experimenta las tecnologías y mide su potencial basado en el conocimiento del área que él tiene y en el conocimiento de las dificultades que entraña para un estudiante la asimilación de estos conceptos, producto también de la experiencia y conocimiento que le ha dado el ejercicio docente a lo largo de su vida profesional "y también con (Díaz Pizón, 2018) cuando alega "No obstante, se debe tener en claro que si bien la 
tecnología educativa es un elemento importante para mejorar los procesos de enseñanza aprendizaje, esta mejora no depende solamente de la utilización de un software educativo, sino de su adecuada integración curricular, es decir, del entorno educativo diseñado por el docente." O sea, la utilización de las TIC como una herramienta tecnológica significa además de una transformación pedagógica entender que las mismas son mecanismos de aplicación y no constituyen de por sí el centro del proceso de enseñanza-aprendizaje. Concordamos con Garcés-Prettel et al. (2014) al afirmar que "facilitan los procesos educativos, más no garantizan en sí mismo su eficiencia como lo sustentan, consecuentemente es más que necesario que los docentes no sepan únicamente usar las Tics, sino que aprendan a integrarlas en sus planes y actividades de curso, lo cual presupone no sólo un cambio metodológico, sino también un análisis del modelo pedagógico y una nueva forma de concebir al docente y estudiante en su quehacer dentro y fuera del aula".

En referencia a la integración de las TICs en el proceso de aprendizaje de las matemáticas, existen diversos criterios en función de la utilización de los tipos de herramientas a utilizar para crear ambientes favorables a la tecnología; entre ellos se encuentra el planteamiento de Andee Rubin (2000) quien las divide en 5 categorías: conexiones dinámicas; herramientas avanzadas; comunidades ricas en recursos matemáticos; herramientas de diseño y construcción; y herramientas para explorar complejidad.

A continuación se recogen algunas de las herramientas de las TIC más utilizadas en el proceso de aprendizaje de las matemáticas:

\section{Software aplicados en el área de conocimiento de matemáticas}

- Matlab: Se define como, "un lenguaje de computación técnico de alto nivel y un entorno interactivo para el desarrollo de algoritmos, visualización de datos, análisis de datos y cálculo numérico"- Dentro de sus principales características se encuentra: "herramientas interactivas para la exploración, el diseño y resolución de problemas, funciones matemáticas para álgebra lineal, estadísticas, análisis de Fourier, funciones de gráficos en 2D y 3D para visualizar datos, herramientas para la construcción de interfaces gráficas de usuario (GUI), entre otras".

- Maple: "Es un software de aplicación que permite realizar desde sencillos cálculos matemáticos, pasando por el desarrollo de hojas de cálculo y la enseñanza de conceptos fundamentales, hasta la creación de sofisticados modelos de simulación de alta fidelidad, incluye miles de funciones matemáticas así como una utilidad para la visualización de gráficas matemáticas en 2D y 3D."

- Cabri II Plus: "Es un software de geometría dinámica que permite la construcción en ordenador de figuras geométricas. Entre sus principales características esta aplicación permite desplegar lugares geométricos, de puntos u objetos, de lugares, y también permite las intersecciones con lugares. Cuenta también con una herramienta denominada "ecuación", la cual permite obtener la ecuación de un lugar en el caso de las curvas algebraicas.

Además deben tenerse en cuenta, las aplicaciones que deben emplear los softwares para elaborar los materiales didácticos digitales, como son: Capturadores de video, Camtasia Studio 
y Adobe Captivate; así como las aplicaciones que deben emplear los software para elaborar presentaciones de multimedia, tales como Prezi, Impress y Flash.

Pero, realmente, en el caso que nos ocupa, ¿Qué estrategias pudieran proyectarse que propicien las condiciones para un aprendizaje significativo de las ciencias matemáticas en el contexto universitario, mediante la aplicación de las TIC?.

$\mathrm{Al}$ respecto, y ante todo, deberán integrarse diversas perspectivas metodológicas que conlleven a la construcción de conocimientos, de docentes y estudiantes, que ofrezcan oportunidades de aprendizaje, a partir de un conocimiento previo de las matemáticas, a través del modelo de aprendizaje significativo, cuyos alcances y objetivos sean los de encaminarse hacia el logro de un cambio de actitud en los estudiantes, a través de la aplicación de las Tecnologías de la Información y Comunicación, que favorezca la contextualización y desarrollo de actitudes positivas y críticas hacia las matemáticas por los estudiantes, de manera que elimine o al menos reduzca significativamente, el desinterés y rechazo hacia ella.

Para ello debe elaborarse una estrategia integral, que interrelacione las diferentes fases de implementación conformadas por el proceso de aprendizaje y de implementación de las TIC en las matemáticas, respetando sus propios alcances de desarrollo que permitan articular ambas fases, con la finalidad de obtener un aprendizaje significativo de manera que los estudiantes puedan desarrollar sus propias capacidades creativas que favorezca la formación cognitiva de los mismos, preparándolos para la vida profesional futura.

Gráfico No1. Estrategia integral de aprendizaje significativo en la matemáticas a través de la aplicación de las tic.

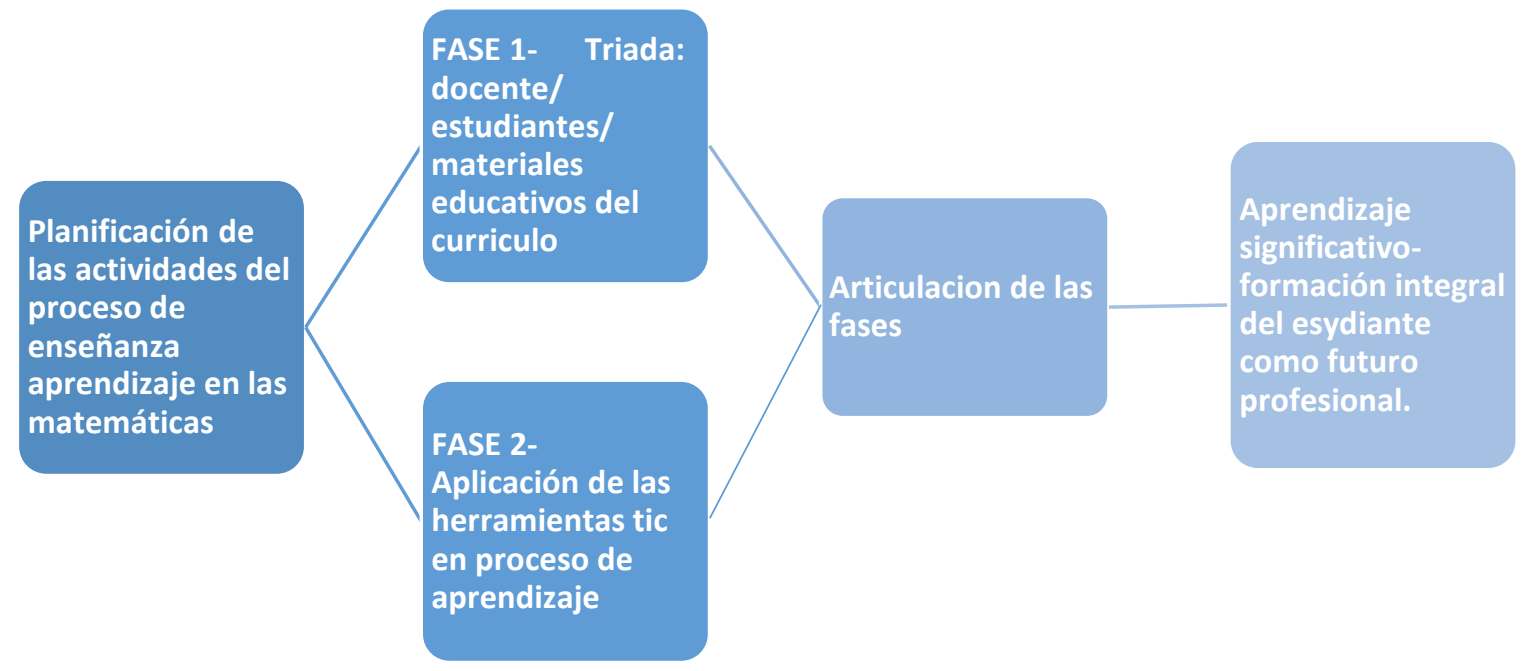

Elaborado por: Grupo de investigación. 


\section{Conclusiones}

- La eliminación del bajo nivel de desempeño, aburrimiento y hasta la apatía hacia el aprendizaje que se producen en los estudiantes con el empleo de métodos tradicionales de enseñanza de las matemáticas, se logra mediante el establecimiento de una relación entre estudiantes-docentes y medios educativos, el currículo y la aplicación de las TICs conducentes a la obtención de un aprendizaje significativo de las matemáticas, que implica un crecimiento cognitivo de los estudiantes y un mayor nivel de motivación por parte de los estudiantes universitarios en esta materia.

- Este logro solo se alcanzará, si se cuenta con un personal docente capacitado, comprometido y consciente, que permitan asimilar los cambios de forma proactiva teniendo como fundamento que la utilización de las TIC como una herramienta tecnológica significa además de una transformación pedagógica entender que las mismas, son mecanismos de aplicación y no constituyen de por sí el centro del proceso de enseñanza-aprendizaje.

\section{Referencias Bibliográficas}

Ausubel, D. P. (1976). Psicología educativa. Un punto de vista cognoscitivo. México: Ed. Trillas.

Andee Rubin. (2000), "Technology Meets Math Education: Envisioning A Practical Future", Julio de 2000. http://www.air.org/forum/abRubin.htm

Chiluiza García, K. (2004). Las TIC y su impacto en las estrategias de enseñanza en escuelas primarias ecuatorianas, Revista Tecnológica, 1(1)

Díaz Pizón, J. E. (2018). Aprendizaje de las matemáticas con el uso de simulación. Sophia 14 (1), 22- 30.

Garcés-Prettel, M., Cantillo, R. R., y Ávila, D. M. (2014). Transformación pedagógica mediada por tecnologías de la información y la comunicación (TIC). Saber, Ciencia y Libertad, 9(2), 217-228

Gowin, D. B. (1981). Educating. Ithaca, N.Y.: Cornell University Press

Grisales, A.M., (2018), Uso de recursos TIC en la enseñanza de las matemáticas: retos y perspectivas. Entramado vol.14, No .2 Julio-Diciembre de 2018, p.198-214 (ISSN 19003803 / e-ISSN 2539-0279). https://orcid.org/0000-0002-4385-4474

Fainholc, B. Una tecnolog'ia educativa apropiada y cr'1tica. Editorial Lumen. Buenos aires, Argentina. 2012.

Landeau, R. (2012) .Metodología y nuevas tecnologías. Editorial Alfa. Caracas, Venezuela

Moreira, M. A. (2012). ¿Al final, qué es aprendizaje significativo? Qurriculum, No 25, Tenerife: Universidad de La Laguna. Servicio de Publicaciones. (En prensa). 
Pérez, R. (2001). Didáctica universitaria y recursos tecnológicos. El proceso de enseñanzaaprendizaje universitario. Universidad Nacional de Educación a Distancia. Vicerrectorado de Calidad e Innovación, 1-26.

Rodríguez Palmero, M. L. (2008). La Teoría del Aprendizaje Significativo. En Rodríguez Palmero, M. L. (org.): La Teoría del Aprendizaje Significativo en la perspectiva de la Psicología Cognitiva. Barcelona: Ed. Octaedro. Págs. 7-45.

Rodríguez Palmero, M. L., Caballero Sahelices, C. y Moreira, M. A. (2010). La teoría del aprendizaje significativo: un referente aún actual para la formación del profesorado. Actas del I Congreso Internacional Reinventar la formación docente. Universidad de Málaga. Págs. 589-603.

Rodríguez Palmero, M. L. (2004 a). La Teoría del Aprendizaje Significativo. Ponencia presentada en la First International Conference on Concept Mapping. Pamplona (España), 14-17 de septiembre. Págs. 535-544.

Rodríguez Palmero, M. L. (2004 b). Aprendizaje significativo e interacción personal. En Moreira, M. A., Caballero Sahelices, C. y Rodríguez Palmero, M. L. Aprendizaje Significativo: Interacción personal, Progresividad y Lenguaje. Universidad de Burgos. Servicio de Publicaciones. Págs. 15-46.

Triana-Muñoz, Mónica María; Ceballos-Londoño, Juan Fernando; Villa-Ochoa, Jhony Alexander. Una dimensión didáctica y conceptual de un instrumento para la Valoración de Objetos Virtuales de Aprendizaje. El caso de las fracciones. En: Entramado. Julio Diciembre, 2016 vol. 12, no. 2, p. 166-186. Disponible en http:// revistasojs.unilibrecali.edu.co/index.php/entramado/article/view/368.

Vega Vega, Juan Carlos; NIÑO DUARTE, Franklin; CÁRDENA, Yini Paola. Enseñanza de las matemáticas básicas en un entorno e-Learning: un estudio de caso de la Universidad Manuela Beltrán Virtual. En: Revista Escuela de Administración de Negocios, 2015, no.

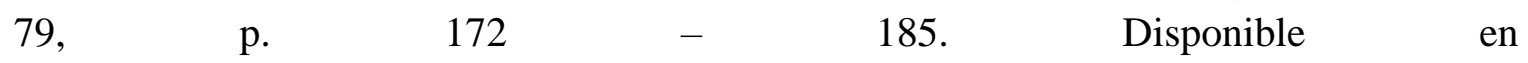
http://www.scielo.org.co/scielo.php?script=sci_arttext\&pid=S0120-81602015000200011

Villa Ochoa, Jhony Alexander; Galvis, Jackeline; Sierra, Ruben; Velez, Lida. Integración de tecnologías en el aula de clase. El caso de los profesores implicados en el proyecto Teso. En: Tecnologías Digitais en Educação: perspectivas teóricas e metodológicas sobre formação e prática docente. Curitiba: Editora CRV. 2014, p. 35-56.

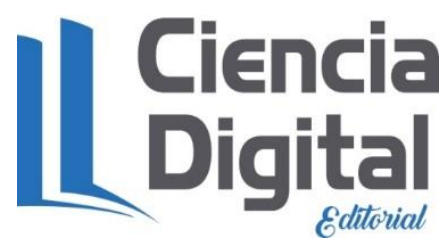




\section{PARA CITAR EL ARTÍCULO INDEXADO.}

Gavilanes Sagñay, M. A., Yanza Chávez, W. G., Colcha Ortiz, A. R., \& Velasteguí López, E. (2020). Tecnologías de la información y la comunicación: bases del aprendizaje significativo en matemáticas en estudiantes universitarios. Explorador Digital, 4(3), 126-139. https://doi.org/10.33262/exploradordigital.v4i3.1301

\section{\Ciencia}

El artículo que se publica es de exclusiva responsabilidad de los autores y no necesariamente reflejan el pensamiento de la Revista Explorador Digital.

El artículo queda en propiedad de la revista y, por tanto, su publicación parcial y/o total en otro medio tiene que ser autorizado por el director de la Revista Explorador Digital.
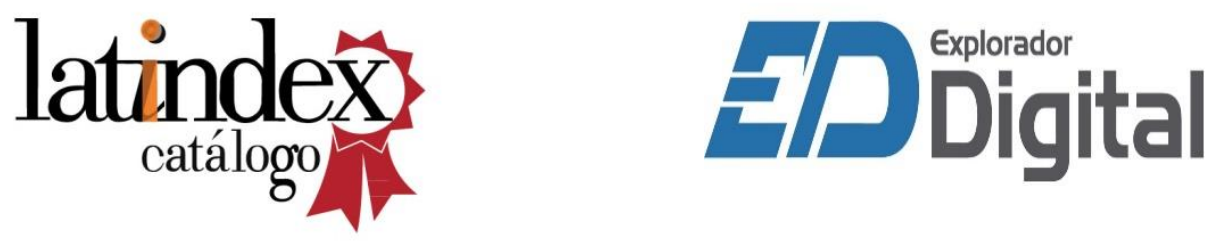